\title{
An Approach based on XML for Communication in Home Healthcare Systems
}

\author{
Javier Martín-Moreno, Daniel Ruiz-Fernández, Member, IEEE, and Antonio Soriano-Payá
}

\begin{abstract}
In this paper we analyze the problems in home healthcare communication and we propose an approach for communication between different technologies implementing a new protocol. We use XML to define the communication frames, stored data and the accessibility. We also propose the ways to access to the information from anywhere using criteria of security, accessibility and the possibility of defining models of actuation before critical situations.
\end{abstract}

\section{INTRODUCTION}

$\mathrm{N}$ OWADAYS, the union of different technologies is the main challenge into $\mathrm{D}_{2} \mathrm{H}_{2}$ (Distributed Diagnosis and Home Healthcare) [1]. The market offers many good solutions to implement an efficient healthcare environment. Systems used at the present days are efficient although they have a fault: they only work with their own technology. When a user buys a new technology to improve his standard of living or to monitor a person, he needs to acquire all the devices which must be compatible with the technology chosen.

We propose an open model of communication for healthcare systems and embedded devices. The model defines a central core to collect the information from the sensors. This core includes the interfaces related to the different sensors present in the environment. We propose the use of new sensors with some special features: lower in consumption, smaller in size and wireless. These devices would use a mesh topology of interconnections, giving the chance of creating multiple ways to send information from the sensors to the central core.

The model implements a DB (database) into XML (eXtensible Markup Language) in order to have an easier access to the data, using the recommendations from W3C for native XML DB [2]-[5].

The model also grants the chance of monitoring the information [6], [7] by different users, placed in different places. Considering that the stored information is private, the model sets four roles into the system with different authorization and level of access: doctors, patients, family and staff. Moreover, it is enabled an easier communication among patient, families and medical staff, by using a web

Javier Martin-Moreno is with the Department of Computer Technology, University of Alicante, SPAIN (phone: (+34)96590-34-00 Ext. 3331; fax: (+34)96590-96-43; e-mail: jmartin@dtic.ua.es).

Daniel Ruiz-Fernandez is with the Department of Computer Technology, University of Alicante, SPAIN (e-mail: druiz@dtic.ua.es).

Antonio Soriano-Paya is with the Department of Computer Technology, University of Alicante, SPAIN (e-mail: asoriano@dtic.ua.es). interface. The patient adds information in the DB of the system specifying any possible change in his disease; this enables doctors to give a better diagnosis or to arrange a meeting with the patient.

In this paper we will be focused into the proposed model, deeping into the components of the communication, the phases of the communication and the analysis of the data transferred. Next we speak about the access to the information method with our proposal of standard language implementation. Finally we will see a scenario where the approach can be used.

\section{COMMUNiCATION MODEL}

The communication process begins in the sensors; the information collected from the patient is sent to the central core. The system analyzes and stores the data so that the information can be available for everybody; implementing an interface with different methods of access. We set for the model three elements in the environment: sensors, central core and external nodes.

The communication has five phases in our model: discovery, translation, answer to the discover/installation, information transfer and installation.

\section{A. Communication Elements}

1) Sensors

Sensors collect data from the patient and his environment. We can found different sensors according to the way of getting data. For example, sometimes it is necessary to collect constantly information from patient; in this case it is desirable to have a small and portable device in order to be comfortable for the patient to wear it. That is the reason why the model proposes the use of sensors with wireless communication system, smaller and lower in consumption.

Several types of sensors can be employed in order to monitor the patient. For example, sensors of biometrical features can be used to identify a user or presence sensors can detect the activity of elderly people.

\section{2) Central Core}

The central core collects the data from sensors, analyzes and stores them. Once the information is stored, it is possible for any authorized user to have access to it. The central core implements four functions:

--Communication. In this phase the central core collects the information sent from the sensors in their particular protocol frame format. 
--Translation. When the data sent by the sensors reach the interface of the system, they are transformed into the XML frame defined by the model.

--Process. The information is analyzed by the system which looks for any peculiar case (emergency). If no problem appears, the data is stored in the XML DB.

--Access to the Information. The last function in the system is to offer the information to the users. Due to the privacy of data, the central core uses a role classification to grant or deny the access to the information.

\section{3) External Nodes}

These nodes are related with the different ways that users have to access to the system in anywhere and in any time. The model employs a standardized system to use from different devices (Computer, PDA, SmartPhone, etc) using a web interface. Due to security rules, the user needs to be authenticated by the system, using his role access level.

To understand more the final configuration of the architecture of the model proposed can be seen in figure 1 .

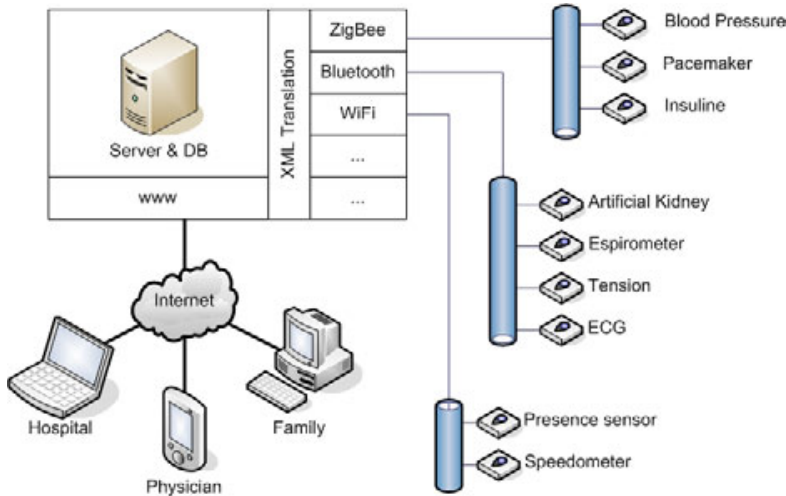

Fig. 1. Architecture of the model proposed. On the right we identify the different sensors and technologies. On the left up we can see the central core with all his parts defined. Finally, on the left down there are three external nodes, accessing from different platforms and operating systems.

\section{4) Connection Topology}

The external nodes connect to the system using Internet, through an IP direction at home and NAT (Network Access Transfer). The model employs mesh topology for internal nodes (sensors and central core). If a device is too distant from the central core, it uses another sensor to transfer the information. If a node fails, the sensor selects another device in the nearby to continue the communication without any loss of data. An example of communication where the way is cut and recovered (adaptive routing system) thanks to the mesh topology is showed in figure 2.
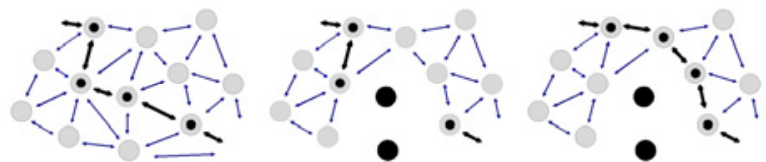

Fig. 2. Adaptive routing system. First figure presents a possible way of communication. Second represents two node fails, breaking the communication way. Third shows the new way.

\section{B. Communication Phases}

--Discover. This phase is the first step for any new device that wants to join to the system or when his network has been loosed. The central core is asked by the sensor to join in the network environment. This frame is able to arrive to the central core directly or using another sensor that implements the same protocol.

--Translation. The phase begins when the information from the sensors arrives to any interface of the central core. In this phase, the frames are translated from the particular protocol format of the sensor to the model standard XML.

--Answer to the Discover/Installation. The central core uses the same frame in two different situations: answer to the discover (which follows the discover phase) and answer to the installation (which follows the phase of installation).

In this phase the central core communicates to the sensor whether it is accepted in the network by the central core or not. After a deny frame, the system saves a fail attempt to access in the DB and it will be analyzed for security reasons.

--Information Transfer. When the discover or the installation phase finishes correctly, the device begins to collect information from the patient and sends it to the central core. The frame used is constituted of two parts: the former consists in the patient's identificative data together with the device, the latter is the information taken from the patient.

--Installation. If the discover phase fails, the sensor must be installed so it begins to send to the central core all the information about itself (identification, signature, etc.). Moreover, in this step, the device sends his driver to the central core to be installed.

\section{ANALYSIS OF THE INFORMATION}

The information used in the environment is private, therefore the model has to implement cryptographic techniques to avoid any non authorized access or modification.

Any frame, which is carrying the patient's information, is encrypted before being sent to the central core, in order to avoid being sniffed. When the central core receives the frame it is decrypted and then it is translated into a XML frame. With the new frame, the system analyzes the information and verifies if it comes from the sensor or not.

In our proposal it is used the AES (Advanced Encryption Standard) to protect the information [8]. This technique uses a matrix with $4 \times 4$ bytes called state to make the process, and in the process it is made four cycle steps, except in last iteration. The steps are Sub Bytes (each byte is replaced by its substitute in state), Shift Rows (bytes are arranged in a rectangle, and shifted), Mix Columns (matrix multiplication) and Add Round Key (a xor operation). In the last iteration the Mix Columns step is removed.

Data received are analysed; if the values are normal, the central core saves the data into the standard XML frames; else, the actuation model of the type is executed. 


\section{A. Data work}

When a new frame arrives, the system accesses to the DB and it obtains information about the sender. This information is necessary for two objectives: to know if the sensor exists into the network and to take the emergency values of the information associated to this sensor. In the next step, the central core tests the data collected by a sensor and analyzes them, determining if any value is out of the normal values or not. If data do not show any abnormality, the central core saves them into the DB. On the contrary, the data are stored considering their abnormal features and the system begins to use the corresponding actuation model.

\section{B. Actuation Model}

The actuation model collects the information and the alert priority from DB. If the information has a priority alert, the system analyzes carefully the frame looking for possible problems. When the central core receives the information, it chooses how to behave: whether doing nothing, sending an email to the doctor or beginning the emergency process sending the information to the hospital.

Even if the system begins an emergency process, the central core continues collecting new information from patient's sensors. The system has separated processes to control alerts and to take data from patient simultaneously. This makes possible to know the state of the patient from the hospital to check the alert and begins the appropriate medical protocol.

\section{DESIGN OF THE INTERFACE}

Physicians, family and patients should have access to the stored information. Therefore, as the data are personal and private, it is necessary to differentiate the access depending on the person who wants to access to the information, using different and not overlapped roles. Furthermore it is needed the access to the information anywhere, this is possible implementing a web interface.

\section{A. Type of users}

The system filters the data depending on the privilege of access, due to the definition of four different kinds of users: physicians, family, patient and service staff.

--Physicians. This group is constituted by physicians and medical staff from a hospital. These users can add new information about a patient into the system to complete the medical history and to give a diagnosis on the basis of the data that had been collected from sensors.

--Family. This group has restricted access to the information concerning the patient. Before showing the data from DB, the system generates basic data that can be available to not specialized people. Anyway, the patient can limit the information that his relatives can see.

--Patient. The monitored patient has access to the information system. It can arrange a meeting with his doctor or any other specialist. It also can add new diseases or symptoms. If the patient needs to use a device without recognition mechanisms, it is possible to indicate which sensor would like to use.

--Maintenance service. These users have the maintenances functions of the network, web service and sensors. They have access to the system to make modifications or reparations but they do not have access to the patient's information.

\section{B. Web Interface}

The access to the information is carried out using web technologies. This implementation offers the chance of access to the information using the same interface from anywhere. This method includes other possibilities like the chance of download the stored information, to analyze them with different applications using the standard XML format. The system has to filter the information using the permissions of the user roles. Moreover, if the patient has any disability and he cannot have access to the web system, it is possible to use adapted interfaces. We propose two new interface modules for deaf or blind people. The first adapted implementation consists in the use of artificial vision to allow the patient uses the interface with his movements. The second implementation uses voice recognition for interact with the interface by saying the instructions.

\section{IMPLEMENTATION OF THE PROTOCOL}

The information follows the standard format in XML to elaborate the communication frames and the stored information in the DB.
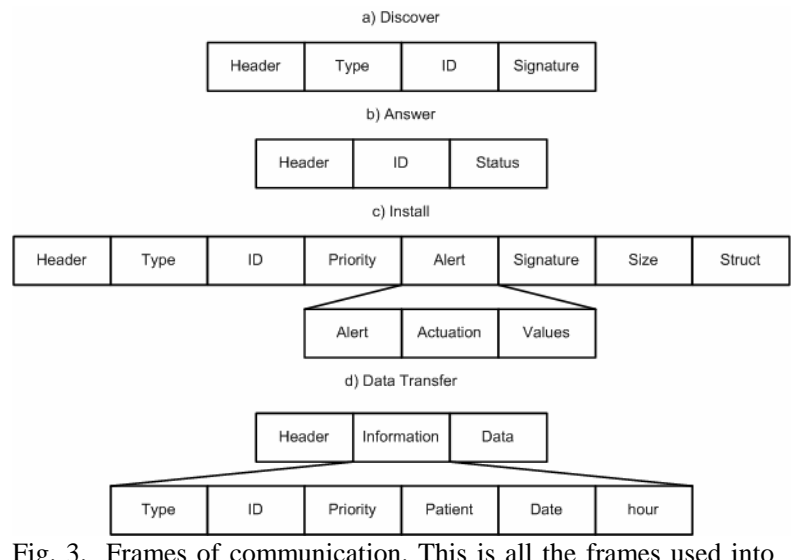

Fig. 3. Frames of communication. This is all the frames used into the communication model.

\section{A. Frame}

The frames (figure 3 ) are defined in the model using the utf-8 codification into the 1.0 XML version. To be more specific it uses XML Schemas, more complex and complete than DTD (Data Type Definition), due to his extensibility, data type specification and the syntax completely into XML.

The model sets a schema specification for every frame, using sequences of complex types in the definition in order to have an easier and more direct analysis. In Code1 we can see an example of a Schema implemented. 


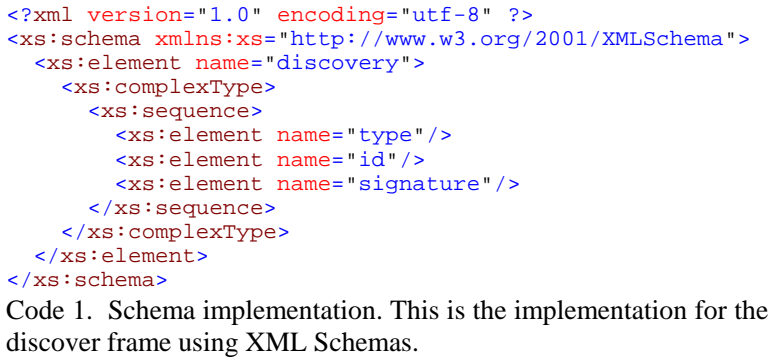

Code 1. Schema implementation. This is the implementation for the discover frame using XML Schemas.

\section{B. Native $X M L D B$}

The DB is implemented into XML. The model follows the propositions from W3C (World Wide Web Consortium) about access to native XML DB using XPath 2.0 (for scan and process XML documents), XQuery 1.0 (for query collections of XML data) and XSLT 2.0 (for transform XML documents into other document format and in a different XML document).

The model organizes the DB in eleven different schemas implemented together into the system: devices; type (to store the type of devices); emergency (with the actuation protocols for emergency cases); users; patients; diseases and illnesses; medicine (to store the treatment for the illnesses); physician; data; hospital; deny devices. The relations between the schemas in the model (figure 4) are: a patient is related to a hospital, a physician and diseases. A disease has a list of medicines or drugs for the treatment. The patient can have assigned different devices. Devices has a type and alert values. Finally the data depend on a device and a patient.

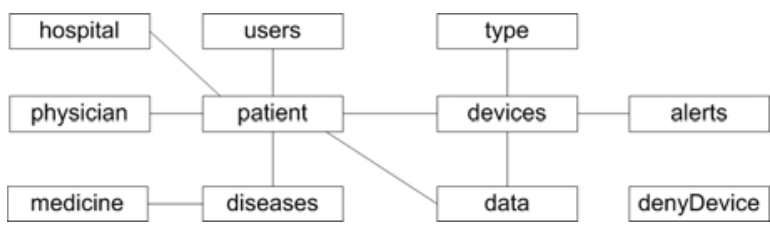

Fig. 4. System DB relations. This diagram represents the relations between the XML documents into the native DB.

\section{SCENARIO}

The work proposed in this paper can be used in many different scenarios; for example we can think in a house in which we put three different types of sensors: step counter, presence sensors and accelerometer. The devices and the central core make a mesh network.

With the step counter information it is possible to know the daily activity of the patient. To know in which room the patient is, we use the presence sensor information. And using the accelerometer it is possible for the central core to know if the patient has fallen. Working with the three types of sensors together it is possible, for example, to know if an elderly person goes to the kitchen very frequently but he does not remember it: this can be the beginning of Alzheimer's disease.

\section{CONCLUSION}

In this article we have proposed a system to make independent a home healthcare system from the technology of the sensors used. We define a protocol based on XML which uses four main frames for the communication. We also use XML to store all the information into plain text documents. The use of a XML DB has advantages to access and get the data stored, because it is not needed to make any format translation.

The system proposes includes some control process that makes possible the definition of actuation models, oriented to danger or risk situations. Many new possibilities appears from this environment; the patient can be monitored every time, including when is out of home. It is possible to use portable devices (e.g. a PDA or mobile) in the exterior, taking information and synchronizing with the central core when the patient arrives to home. Furthermore the system can warn someone automatically in an emergency.

The model is opened enough to offer the possibility of adding actuators to the system so it would be possible to solve emergencies as for example to inform the patient that it is time to take a pill or to change the saline solution for an artificial kidney.

\section{REFERENCES}

[1] Prabaharan, K., Lavanya, J., Goh, K.W., Kim, Y., Soh, C.B "Distributed Architecture towards Tele-Diagnosis" Distributed Diagnosis and Home Healthcare, 2006. $\mathrm{D}_{2} \mathrm{H}_{2}$. 1st Transdisciplinary Conference on April 2-4, 2006 pp. 105- 108

[2] Sokic, M., Matic, V., Bazant, A "Web content management system based on XML native DB" Information Technology Interfaces, 2003. ITI 2003. Proceedings of the 25th International Conference on 16-19 June 2003 pp 457- 462.

[3] Vakali, A., Catania, B., Maddalena, A "XML data stores: emerging practices” IEEE Internet Computing Volume: 9 Issue: 2 March-April 2005 pp. $62-69$

[4] Pardede, E., Rahayu, W., Taniar, D "Maintaining data consistency in XML-based applications” Industrial Informatics, 2005. INDIN '05. 2005 3rd IEEE International Conference on 10-12 Aug. 2005 pp. 510515

[5] Khin-Myo win, Wee-Keong Ng, Ee-Peng Lim “An architectural framework for native XML data management” Cyberworlds, 2003. Proceedings. 2003 International Conference on 3-5 Dec. 2003 pp. 302- 309.

[6] Tohme, W.G., Inyoung Choi, Vasilescu, E., Mun, S.K. "The Evolution of Distributed Diagnosis: Teleradiology As a Case Study” Distributed Diagnosis and Home Healthcare, 2006. $\mathrm{D}_{2} \mathrm{H}_{2}$. 1st ransdisciplinary Conference on April 2-4, 2006 pp. 113- 115

[7] White, C.C., Fang, D., Eung-Hun Kim, Lober, W.B.; Yongmin Kim "Improving Healthcare Quality through Distributed Diagnosis and Home Healthcare $\mathrm{D}_{2} \mathrm{H}_{2}$ ” Distributed Diagnosis and Home Healthcare, 2006. $\mathrm{D}_{2} \mathrm{H}_{2}$. 1st Transdisciplinary Conference on April 2-4, 2006 pp. 168- 172

[8] Bertoni, G. Bircan, A. Breveglieri, L. Fragneto, P. Macchetti, M. Zaccaria, V. "About the performances of the Advanced Encryption Standard in embedded systems with cache memory" Circuits and Systems, 2003. ISCAS '03. Proceedings of the 2003 International Symposium on Volume 5, 25-28 May 2003 Pages:V-145 - V-148 vol.5 